\title{
Meshless methods with application to Resin Transfer Molding simulation
}

\author{
J.A. García`, Ll. Gascón*, E. Cueto*, F. Sánchez`, F. Chinesta^ \\ Universidad Politécnica de Valencia. Dpto Ing. Mecánica y Materiales, Camino Vera s/n, 46022, \\ Valencia (Spain).jugarcia@mcm.upv.es \\ (\$) Universidad Politécnica de Valencia. Dpto Matemática Aplicada, Camino Vera s/n, 46022, \\ Valencia (Spain).11gascon@mat.upv.es \\ (*) Aragón Institute of Engineering Research (I3A), University of Zaragoza, María de Luna 3, E-50018 \\ Zaragoza(Spain).ecueto@unizar.es \\ ( ) Universidad Cardenal Herrera CEU. Escuela Superior de Enseñanzas Técnicas C/ San Bartolomé \\ 55, E-46115 Alfara del Patriarca, Valencia (Spain). sanchezf@uch.ceu.es \\ ( ^) Laboratoire de Mécanique des Systèmes et des Procédés - ENSAM Paris, 151 Boulevard de \\ l'Hôpital, F-75013 Paris (France).francisco.chinesta@paris.ensam.fr
}

\begin{abstract}
In this work it is studied and analyzed the possible advantages to simulate the mold filling process in Resin Transfer Molding (RTM) with an updated Lagrangian formulation. For this purpose, the well known meshless natural element method (NEM) [1] was implemented to simulate the mold filling process. In this approach, nodes are distributed in the calculation domain, however no mesh is needed to interpolate the unknown functions. This technique presents some advantages over classical finite element simulations (FEM): (1) no remeshing of the transient saturated domain is required at each calculation step; (2) the accuracy of the interpolation is not significantly affected by the nodal distribution. The use of meshless techniques also prevents from having to cope with the numerical instabilities generally associated with the numerical resolution of the transport equations that arise in RTM [2], such as in the heat balance during mold filling or in the equations giving the position of the fluid front or the incubation time at each calculation step. In this paper, the position of the flow front and the geometry of the fluid saturated domain in the mold cavity are handled by invoking the geometrical concept of $\alpha$-shape for a cloud of nodes [3,4], which allows to extract the shape of the domain being simulated by employing a cloud of nodes only. Numerical examples are presented to show the performance of the proposed new numerical formulation.
\end{abstract}

Keywords: Resin Transfer Molding, Liquid Injection Molding, Meshless, Natural Element Method, $\alpha$-shape.

PACS: $81.05 . \mathrm{Qk}$.

\section{INTRODUCTION}

Injection strategy is a practical issue in RTM in which the resin can be injected into the mold using multiple gates. Traditionally the selection of gate and vent locations in mold design has been based on the designer knowledge and trial and error attempts, but many recent research studies have been conducted to optimize the process by using computer simulation. The most important task in simulation of the mold filling 
process is the determination of the location of the moving free surface defined by the liquid resin flow front. Methods employed to solve such problems can be classified into two groups: Lagrangian and Eulerian schemes. The Eulerian methods use a fixed grid system, which is generated over the entire domain and is not changed until the completion of calculation. However, a special treatment is neccesary to track the moving free surface because the motion of fluid does not coincide with the calculation mesh. Fluid flows in porous media have been succesfully solved by an approach based on finite element method. In general, two alternative Eulerian numerical techniques using a fixed mesh have been developed for accounting moving interfaces in RTM: the Volume of Fluid techniques [5] and the level set methods [6]. However, the shape of the flow front is not necessarilly accurate by these numerical methods. Mesh adaptivity techniques have been impemented to overcome this issues in RTM modeling [7], but in these problems accurate finite element solutions require usually remeshing for each new position of the flow front, which makes the simulation quite expensive in terms of computer time.

Lagrangian schemes are characterized by the mesh system, which is moved or deformed as the calculation proceeds. In the last years, a wide family of computacional methods has emerged in a Lagrangian framework. These methods have been used by many researchers for treating a large variety of engineering problems. All of them share the characteristic of no need of explicit connectivity information, which is built by the method in a process transparent to the user. Many of these meshless methods do not interpolate nodal values and, as a consequence, do not exactly reproduce essential boundary conditions. This and other problems, make their use not widely applicable and have demanded the attention of many researchers in the last years. The Natural Element Method (NEM) [1] is one of the methods based on a Galerkin implementation, built upon the so-called natural neighbor interpolation. This interpolation scheme is based on the concepts of Voronoi diagrams and Delaunay triangulations, and it has very interesting features, like its strictly interpolant character and its ability to exactly interpolate piece-wise linear boundary conditions.

In this paper, we propose the use of the NEM to model the flow front position of the injected resin in the RTM process. First, the NEM is reviewed. Then, the governing equations and boundary conditions for the RTM flow model are presented. Finally, we present some numerical results which show the interest of the NEM to simulate the filling process in the context of a simple RTM case. In this case, the position of the flow front and the geometry of the fluid saturated domain in the mold cavity are handled by invoking the geometrical concept of $\alpha$-shape for a cloud of nodes $[3,4]$, which allows to extract the shape of the domain being simulated by employing a cloud of nodes only.

\section{NUMERICAL IMPLEMENTATION}

In this section we describe the numerical model of the RTM process in the context of a natural neighbour Galerkin method. We review the governing equations and the boundary conditions. Then we describe the technique used to deal with the flow front and the geometry of the fluid saturated domain. 


\section{The Natural Element Method}

The NEM interpolant is constructed on the basis of the Voronoi diagrams of a nodal set and its dual, the Delaunay triangulation. A first-order Voronoi diagram for a set of nodes $N=\left\{n_{1}, n_{2}, \ldots, n_{m}\right\}$ in $\mathbb{R}^{2}$, with coordinates $\left\{x_{1}, x_{2}, \ldots, x_{m}\right\}$ is a subdivision of the space in regions such that any point in $T_{i}$ is closer to the node $n_{i}$ than to any other in $N$. Two nodes whose associated Voronoi cells share an edge are called natural neighbours. The Sibson coordinates of a point $x$ with respect to a natural neighbor $n_{i}$ is defined as the ratio of the overlap area of $T_{i}$ to the total area of the Voronoi cell related to point $x$ :

$$
\phi_{i}(x)=\frac{A_{i}(x)}{A(x)} \quad \text { with } \quad A(x)=\sum_{j=1}^{n} A_{j}(x)
$$

When the point $x$ coincides with the node $n_{i}$, i.e., $x=x_{i}, \phi_{i}\left(x_{i}\right)=1$, and all other shape functions are zero, i.e., $\phi_{j}\left(x_{i}\right)=\delta_{i j}$. In consequence, the natural neighbor interpolant has the important properties of positivity, interpolation and partition of unity [1]. The linear consistency of the interpolant is derived after the local coordinate property

$$
x=\sum_{i=1}^{n} \phi_{i}(x) x_{i}
$$

in conjunction with the partition of unity property [1]. Another property of this interpolants is the ability to reproduce linear functions over the boundary of convex domains [1].This is not true in the case of non-convex boundaries, then a suitable method to enforce essential boundary conditions is necessary. Among different possibilities, we use the geometrical concept of $\alpha$-shape for a cloud of nodes [3, 4], which allows to extract the shape of the fluid saturated domain in the mold cavity being simulated and the position of the flow front by employing a cloud of nodes only. For a more discussion on the concept of $\alpha$-shape and its application for modelling nonconvex domains, the interested reader can refer to [4] and the references therein.

Finally, Natural neighbor shape functions are $C^{\infty}$ at any point except at the nodes and across the interface, where it is $C^{0}$, which allows an accurate treatment of models involving moving interfaces, as the calculation of flow front advance in RTM. The above properties make natural neighbour Galerkin methods an attractive choice among the wide field of meshless methods. The key difference between the formulation of the NEM method upon FEM techniques is the computation of shape functions. For the NEM method, the trial and test functions are constructed using the before natural neighbor coordinates. Then, a standard Galerkin procedure is used to obtain the discrete system which is solved for the unknown vector. 


\section{Governing Equations}

The governing equations which describe the RTM process are given by Darcy's law

$$
\underline{v}=-\frac{\underline{\underline{k}}}{\mu} \nabla p
$$

and the incompressibility equation, expressed as

$$
\operatorname{div} \underline{v}=0
$$

where $\underline{v}$ is the velocity, $\underline{\underline{k}}$ is the preform permeability tensor, $\mu$ is the fluid viscosity and $p$ is the pressure. The fluid flow problem is defined in a volume $\Omega$,

$$
\Omega=\Omega_{f}(t) \cup \Omega_{e}(t)
$$

where the fluid at time $t$ occupies the volume $\Omega_{f}(t)$. The boundary conditions to impose in $\Omega_{f}(t)$ are:

- The pressure gradient in the normal direction to the mold walls is zero.

- The pressure or the flow rate is specified at the injection nozzle.

- Zero pressure is applied on the flow front.

Assuming constant permeability and viscosity, orthotropic preform and the fluid incompressibility, then the variational formulation of Darcy's law extended to the fluid domain yields

$$
\int_{\Omega_{f}(t)}\left(\nabla p^{*} \underline{\underline{k}} \nabla p\right) d \Omega=0
$$

The numerical resolution of the governing equations will be performed by means of a NEM Galerkin technique. Then the resolution scheme is based in solving until the complete filling of the mold the next steps:

1. Obtain the pressure field using a NEM discretisation of the variational formulation given by Eq.(6). The velocity vectors are interpolated using the Sibson shape functions, and can be written in the form

$$
p(x)=\sum_{j=1}^{N} \phi_{j}(x) p_{j}
$$

2. Compute the velocity field from Darcy's law.

3. With the flow kinematics known at time $t_{n}$, i.e., being velocities and pressures at time $t_{n}$ known, $\underline{v}_{n}$ and $p_{n}$ respectively, the position of nodes can be updated at the same time that the flow front position,

$$
x_{i}^{n+1}=x_{i}^{n}+v_{i}^{n} \Delta t
$$

4. Introduce new nodes.

5. Update the fluid domain.

\section{Numerical Results}

In order to validate the proposed NEM technique for RTM process, a flat geometry mold with an obstacle has been simulated, as is shown in Figure 1. The mold is 1 meter in long and 0.5 in width. The injection pressure is 2 bars, the perform permeability is $1 e-8 m^{2}$ and the resin viscosity is $0.1 \mathrm{pa}^{*}$. At the beginning of the filling process, a uniform node distribution in the injection nozzle has been 
considered. These nodes will be moved during the injection and define the filled domain of the mold. Due to these nodes will be more separated at each time step, it is necessary to introduce new nodes in the domain. In this work have been used two different strategies: add nodes in the injection nozzle and on the melt front. The mold geometry is defined by a set of nodes located at the mold boundary. Obviously, all fluid domains nodes must be enclosed by the contour defined by the boundaries nodes.

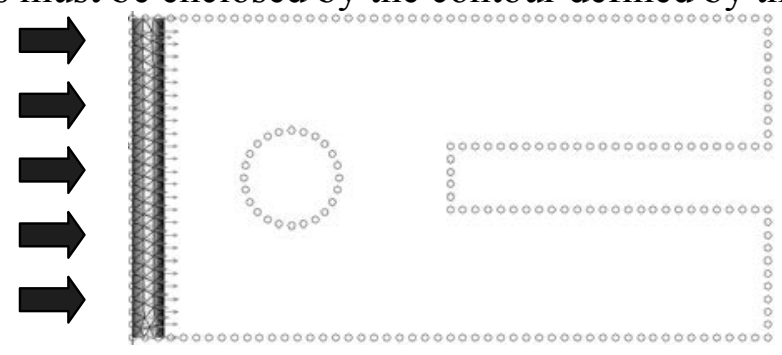

FIGURE 1. Mold geometry and injection conditions.

In the case of considering that the nodes are introduced through the injection nozzle, the position of each one is exactly the node path inside the mold, and then we can track easily any property of the fluid. Usually these kinds of properties require an accurate treatment of the advection equation governing these evolutions. The incubation time constitutes an example of those kinds of properties. This strategy has the disadvantage that the position of all the nodes must be updated at each time step, if we consider a constant time step, we will obtain a greater nodes density near the injection nozzle. Also it is possible that the nodes remain accumulated in certain parts of the mold, depending on its geometry.

On the other hand, the strategy of introducing the nodes in the melt front presents the advantage that only it is necessary update the positions of the new nodes, then it is easier to control the nodes density. However, the physical interpretation of the results is sometimes more complex since the position of the nodes indicates the position of the melt front at different time instants. At each time step the position of the nodes located on the melt front is checked. The time step must be small enough to avoid that nodes move out of the mold. When the distance between the boundaries nodes ant the melt front nodes is small enough, these nodes are activated as bodes belonging to the fluid domain, as is represented in Figure 2.

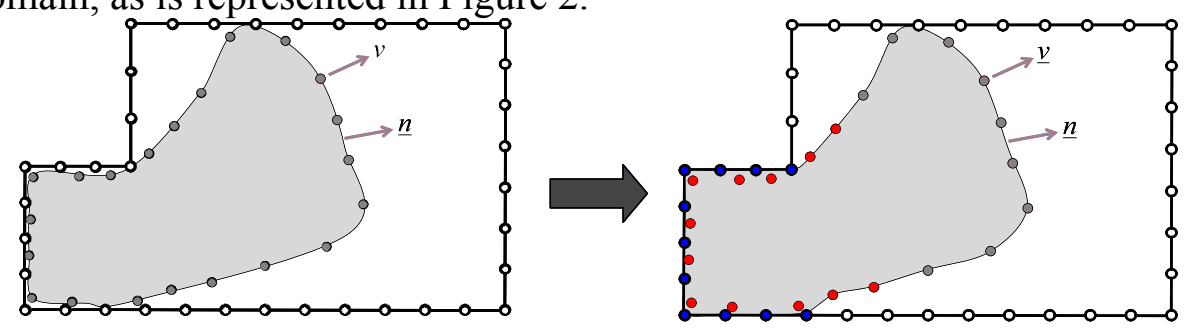

FIGURE 2. Boundary domain adjustment during the injection

The boundary nodes belong either to the melt front or to the mold boundary. At the nodes located on the melt front the null pressure condition applies. Figure 3 shows a sequence of a mold filling. 

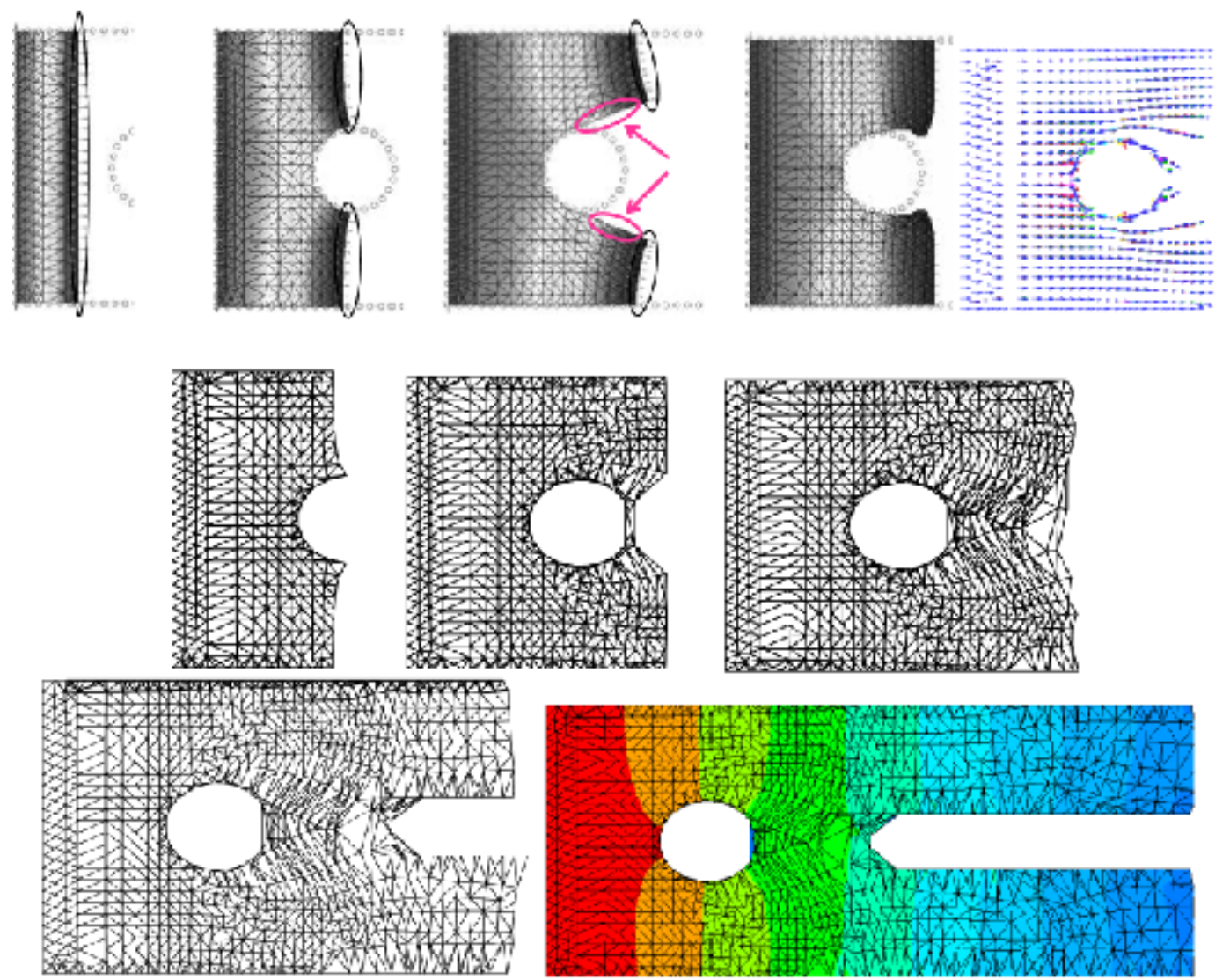

FIGURE 3. Mold filling simulation

\section{ACKNOWLEDGMENTS}

This research work is supported by a grant from Ministerio de Ciencia y Tecnología (Spain), Proyect DPI2004-03152 and Generalitat Valenciana under GVA05/209.

\section{REFERENCES}

1. N. Sukumar, B. Moran, T. Belytschko, "The natural element method in solid mechanics", Int. J. Numer. Methods Engrg. 43 (5), 839-887 (1998).

2. F. Sánchez, J.A. García, Ll. Gascón, F. Chinesta, "Towards an efficient numerical treatment of the transport problems in the Resin Transfer Molding Simulation", accepted for publication in Comput. Methods Appl. Mech. Engrg. (2006).

3. M.A. Martínez, E. Cueto, I. Alfaro, M. Doblaré, F. Chinesta, "Updated Lagrangian free surface flow simulations with natural neighbour Galerkin methods", Int. J. Numer. Methods Engrg. 60, $2105-$ 2129 (2004).

4. I. Alfaro, J.Yvonnet, E. Cueto, F. Chinesta, M. Doblaré, "Meshless methods with applications to metal forming", Comput. Methods Appl. Mech. Engrg. 195, 6661-6675 (2006).

5. C. W. Hirt, B. D. Nichols, "Volume-Of-Fluid (VOF) Method for the Dynamics of Free Boundaries", J. Comput. Phys. 39, 201-225 (1981).

6. J. A.Sethian, P. Smereka, "Level Set Methods for Fluid Interfaces", Annu. Rev. Fluid Mech. 35, 341-372 (2003).

7. E. Bechet, E. Ruiz, F. Troucho, JC. Cuillere, "Remeshing algorithms applied to mould filling simulations in resin transfer moulding”, J. Reinf Plast. Compos. 23(1), 17-36 (2004). 